\title{
Correlation of a scanning laser derived oedema index and visual function following grid laser treatment for diabetic macular oedema
}

\author{
C Hudson, J G Flanagan, G S Turner, H C Chen, L B Young, D McLeod
}

Br J Ophthalmol 2003;87:455-461

See end of article for authors' affiliations

Correspondence to: Chris Hudson PhD, School of Optometry, University of Waterloo, Waterloo, Ontario N2L 3G1,

Canada; chudson@

sciborg.uwaterloo.ca

Accepted for publication 11 September 2002

\begin{abstract}
Aim: To correlate change of an oedema index derived by scanning laser tomography with change of visual function in patients undergoing grid laser photocoagulation for clinically significant diabetic macular oedema (DMO).

Methods: The sample comprised 24 diabetic patients with retinal thickening within $500 \mu \mathrm{m}$ of the fovea. Inclusion criteria included a logMAR visual acuity of 0.25 , or better. Patients were assessed twice before a single session of grid laser treatment and within 1 week of, and at 1, 2, 4, and 12 weeks after, treatment. At each visit, patients underwent logMAR visual acuity, conventional and short wavelength automated perimetry (SWAP), and scanning laser tomography. Each visual function parameter was correlated with the mean oedema index. The mean oedema index represented the z-profile signal width divided by the maximum reflectance intensity (arbitrary units). A Pearson correlation coefficient (Bonferroni corrected) was undertaken on the data set of each patient.

Results: 13 patients exhibited significant correlation of the mean oedema index and at least one measure of visual function for the $10^{\circ} \times 10^{\circ}$ scan field while 10 patients correlated for the $20^{\circ} \times 20^{\circ}$ scan field. Seven patients demonstrated correlation for both scan fields. Laser photocoagulation typically resulted in an immediate loss of perimetric sensitivity whereas the oedema index changed over a period of weeks. Localised oedema did not impact upon visual acuity or letter contrast sensitivity when situated extrafoveally.

Conclusions: Correlation of change of the oedema index and of visual function following grid laser photocoagulation was not found in all patients. An absence of correlation can be explained by the localised distribution of DMO in this sample of patients, as well as by differences in the time course of change of the oedema index and visual function. The study has objectively documented change in the magnitude and distribution of DMO following grid laser treatment and has established the relation of this change to the change in visual function.
\end{abstract}

M acular oedema is the leading cause of visual impairment and legal blindness in diabetic patients. ${ }^{1-3}$ The assessment of diabetic macular oedema (DMO), using stereo fundus biomicroscopy, relies upon the subjective interpretation of retinal thickening. ${ }^{45}$ Early retinal thickening is difficult to differentiate from normal between subject variation in retinal thickness. ${ }^{6}$ Consequently, the detection of change in retinal thickness over time is problematic. Indeed, substantial differences have been demonstrated to exist between experienced medical retina specialists when defining the position and extent of retinal thickening (using stereo fundus biomicroscopy) for a given patient. ${ }^{7}$ The development of a reproducible, objective technique to identify retinal thickening, and to monitor change in thickening, is necessary for the clinical management of DMO and for the evaluation of the efficacy of grid laser photocoagulation or new therapeutic protocols. $^{89}$

The authors have developed psychophysical and objective imaging techniques with the overall aim of improving the clinical management of DMO. Blue on yellow perimetry has been demonstrated to provide improved sensitivity for the detection of DMO when compared to conventional perimetry or visual acuity. ${ }^{10}$ The position of the blue on yellow localised field loss was found to correspond with the area of DMO. Furthermore, grid laser photocoagulation for clinically significant DMO invariably results in a localised loss of white on white and blue on yellow perimetric sensitivity within $10^{\circ}$ eccentricity of the fovea. ${ }^{11}$ Typically, the post-laser loss of perimetric sensitivity was most pronounced immediately after treatment-that is, there was a relative recovery of perimetric sensitivity at 3 months post-treatment (when compared to sensitivity immediately post-laser). Recent work has shown that blue on yellow perimetry can predict the subsequent development of clinically significant DMO in patients with early macular oedema that does not meet ETDRS treatment criteria. ${ }^{12}$ These studies demonstrate that the impact of DMO, and the effect of laser photocoagulation, on visual function is poorly documented and understood.

The analysis of the z-profile signal width of a scanning laser tomographer (SLT) - that is, the width at 50\% maximum reflectance of a function describing change in reflectance intensity plotted against scan depth, has been shown to provide an objective, topographic, and reproducible index of retinal oedema in three selected patients with macular retinal thickening. ${ }^{13}$ The z-profile signal width values of the patients with macular retinal thickening were significantly higher than those of 14 normal subjects. ${ }^{13}$ Other investigators have shown that the normal human Z-profile is narrow and symmetrically distributed at the fovea but is asymmetrically broadened in other macular locations where the retina is thicker..$^{14}$ This basic methodology has been further developed. Empirical observation of SLT images has demonstrated that the maximum reflectance intensity exhibits a localised decrease within areas of DMO relative to the reflectance values of surrounding pixels. ${ }^{13}{ }^{15}{ }^{16} \mathrm{An}$ "oedema index" has been derived that is sensitive to both the localised increase in z-profile signal width and the localised decrease of maximum reflectance intensity. ${ }^{15}$ Using the oedema index methodology (see Methods for 
detailed explanation), oedema maps are generated which illustrate the extent and topographic magnitude of oedema across the SLT image. Note that the term "oedema" is used rather than "thickening" since the analysis incorporates two aspects of the effect of oedema on the z-profile. The oedema index analysis accentuates the presence of oedema rather than simply analysing the effect of retinal thickening. The oedema mapping technique is performed on an individualised basis and it has the advantage of being independent of a reference plane. $^{15}$

The aim of the study was to determine correlation, if any, between change of the oedema index with change of visual function over the 3 month time course of the study for patients undergoing grid laser photocoagulation for clinically significant DMO. Elucidation of the relation between the oedema index and visual function will improve the understanding of the effects of laser photocoagulation on retinal morphology and vision.

\section{PATIENTS AND METHODS \\ Sample}

The sample comprised 24 patients ( 18 males and six females) with clinically significant DMO as defined by the Early Treatment Diabetic Retinopathy Study criteria. ${ }^{17}$ All diabetic patients exhibited oedematous maculopathy (with or without some non-circinate exudate) that was of non-uniform thickness across the macula. Two medical retina specialists independently confirmed the diagnosis of clinically significant DMO using stereo fundus biomicroscopy before inclusion of the patient into the study. The research followed the tenets of the Declaration of Helsinki. Informed consent was obtained from all subjects after explanation of the nature and possible consequences of the study. The study received approval from the Central Manchester research ethics committee. The mean age of the diabetic patients was 59.75 years (SD 8.05 years, range 45-75 years) and the mean time from diagnosis of diabetes was 8.39 years (SD 4.89 years, range $2-18$ years). Three diabetic patients were insulin dependent and 21 patients were receiving oral hypoglycaemic medication. One eye was randomly assigned to the study if both eyes exhibited clinically significant DMO; 11 right eyes and 13 left eyes were assessed. A conservative standardised effect size of 1.0 (based upon the level of variance of the oedema mapping technique in diabetic subjects) was employed for the sample size estimation - that is, the standard deviation of the oedema index was assumed to be equal to the expected effect size. Using a two tailed $\alpha$ of 0.05 and a $\beta$ of 0.10 (that is, power $=$ $90 \%$ ), then the minimum sample size for the diabetic patient group was calculated to be 21 .

Inclusion criteria for all patients included a logMAR visual acuity of 0.25 or better using the $96 \%$ contrast Regan chart (that is, Snellen equivalent visual acuity of 20/30). Exclusion criteria comprised: (i) a distance refractive error greater than plus or minus 6.00 dioptres sphere and/or greater than plus or minus 1.50 dioptres cylinder; (ii) a family history of glaucoma in a first degree relative; (iii) an intraocular pressure $\geqslant 22 \mathrm{~mm}$ $\mathrm{Hg}$; (iv) any other eye disease or disorder (including significant lenticular opacity, see below); (v) any previous laser photocoagulation treatment; (vi) proliferative diabetic retinopathy and its sequelae-for example, vitreous haemorrhage and retinal detachment; (vii) central nervous system (CNS) disorders or psychiatric illness; and (viii) systemic medication with known CNS effects-for example, tranquillisers. Lenticular opacity was graded on the basis of slit lamp appearance and the Lens Opacities Classification System (LOCS) III. ${ }^{18}$ Eyes with the following LOCS III grades were excluded from the study: (I) nuclear colour > NC2; (ii) nuclear opalescence > $\mathrm{NO}_{2}$; (iii) cortical cataract $>\mathrm{C} 2$; and (iv) posterior subcapsular cataract $\geqslant \mathrm{Pl}$.

\section{Visits and treatment}

Diabetic patients were assessed twice before laser treatment (mean interval between pre-laser visits of 12 days, range 1-31 days) and within 1 week of, and at 1, 2, 4, and 12 weeks after treatment. The results of the visual function tests from the first visit were ignored to minimise learning effects. ${ }^{19}$ All diabetic patients underwent a single session of argon green (514 $\mathrm{nm})$, grid laser photocoagulation. The sample represented a wide range of treatment parameters: (i) ablation spot size 50-200 $\mu \mathrm{m}$; (ii) duration of ablation $0.10-0.25$ seconds; (iii) power setting $0.07-0.40 \mathrm{~W}$; and (iv) number of ablations 13-309. The grid laser was applied to the areas of retinal thickening (rather than to the whole macular area as would be appropriate for generalised oedema), as defined by stereo fundus biomicroscopy. Precise details of the treatment for each patient have been reported elsewhere. ${ }^{11}$ Care was taken to ensure that laser burns were not placed at the margin of, or within, the foveal avascular zone.

\section{Procedures}

At each visit, patients underwent $\log$ MAR visual acuity, conventional and short wavelength automated perimetry (SWAP), and SLT in the study eye. The assessment of visual function was always completed before scanning laser tomography. The refractive error was measured at each session before visual acuity assessment and automated perimetry to compensate for any transient refractive changes associated with diabetes. ${ }^{20}$ LogMAR visual acuity was assessed using the $96 \%, 25 \%$, and $11 \%$ Weber contrast Regan charts. Using the Regan charts, a $\log$ MAR visual acuity of 0.0 is equivalent to Snellen visual acuity of $6 / 6$, and 1.0 is equivalent to $6 / 60$ respectively. The charts were illuminated to $100 \mathrm{cdm}^{-2}$ and the recommended viewing distance of 3 metres was employed. A "by letter" scoring system was utilised that gave credit to each correct letter. ${ }^{21}$ The order of chart presentation was randomised to minimise any familiarity that patients may gain of the letter composition of the charts. LogMAR visual acuity was assessed because it has previously been utilised as an outcome measure for the treatment of clinically significant DMO. ${ }^{22-25}$

\section{Automated perimetry}

Automated perimetry was undertaken using the Humphrey Field Analyser 640 (HFA, Humphrey Instruments Inc, San Leandro, CA, USA). The standard, full threshold strategy was used throughout the study. For conventional perimetry, a bowl illumination of $10 \mathrm{cdm}^{-2}$ and a Goldmann equivalent size III (angular subtense $0.431^{\circ}$ ) default static stimulus was used. Conventional automated perimetry was undertaken because it represents the clinical "gold standard" method of assessing the visual field. For SWAP (also termed blue on yellow), a yellow (Schott OG530 filter) bowl illumination of $100 \mathrm{cdm}^{-2}$ and a $440 \mathrm{~nm}$ narrow band ( $27 \mathrm{~nm}$ half power band width) blue stimulus was used. ${ }^{26}$ The default static stimulus for SWAP was a Goldmann equivalent size V (angular subtense $1.724^{\circ}$ ). These stimulus parameters have been shown to provide approximately $1 \log$ unit of short wavelength sensitive pathway isolation. ${ }^{26}$

Program 10-2 of the HFA 640 was used which comprised 69 stimulus locations within $10^{\circ}$ eccentricity of the fovea (including foveal threshold). All patients underwent pupil dilation (to a minimum pupil diameter of $4 \mathrm{~mm}$ ) after visual acuity assessment in order to maximise retinal illuminance and ensure adequate adaptation of the medium and long wavelength sensitive pathways (and also to produce even illumination of the fundus during SLT). The distance refractive correction of the study eye, together with the appropriate near addition for the patient's age, was used to correct for a viewing distance of $33 \mathrm{~cm}$. Mandatory rest periods were given at least every 5 minutes during each perimetry session to minimise the influence of fatigue. ${ }^{27}$ The order of stimulus condition 
(that is, conventional or SWAP) at visit 1 was randomly varied between patients and was then systematically alternated between visits. For visit 2, 15 diabetic patients underwent SWAP first while nine patients initially underwent conventional perimetry.

\section{Scanning laser tomography}

The Heidelberg retina tomograph (HRT) (Heidelberg Engineering, Heidelberg, Germany) was used to acquire topographic measurements of the macula of each patient. The HRT has been described in detail elsewhere. ${ }^{13}$ Single two dimensional section images, comprising $256 \times 256$ pixels (each with 8 bit intensity resolution), were recorded at a repetition rate of $20 \mathrm{~Hz}$. Topographic fundus measurement was achieved by scanning 32 section images (termed a series image) along the optical axis over a period of 1.6 seconds. For each point, or pixel, within the image, the resulting plot of reflectance intensity versus scan depth is termed a z-profile. The peak intensity of the z-profile is assumed to indicate the depth of the vitreous/internal limiting membrane interface.

The refractive error and corneal curvature of each patient was entered into the HRT database before image acquisition (and were unchanged over the period of the study). Steady fixation was achieved using a periscope and a $60 \mathrm{~W}$ light placed at a distance of 3 metres that was viewed with the fellow eye-that is, the periscope allowed patients to see around the HRT during the acquisition of macular images. The monitor aiming cross was employed to centre the image frame on the fovea. Seven HRT images were acquired both for the $10^{\circ} \times$ $10^{\circ}$ and the $20^{\circ} \times 20^{\circ}$ scan fields at each visit. Scan depth remained constant throughout repeated HRT image acquisition of a given eye both within and between visits.

\section{Analysis}

Automated perimetry data

Automated perimetry printouts that exhibited false positive or false negative catch trial response rates greater than 33\%, and those that exhibited fixation losses greater than $20 \%$, were excluded from the analysis. The respective perimetry results from the diabetic patients were compared to databases of normal fields. For conventional automated perimetry, the established STATPAC II normal database ${ }^{28}$ of HFA program 10-2 was utilised. For SWAP, normal HFA program 10-2 sensitivity values were derived from the results of 400 normal subjects between the ages of 18 and 84 years (mean age 48 years, SD 17 years) who had undergone SWAP using program 30-2. The details of the derived program 10-2 normal database have been described in detail elsewhere. ${ }^{10}$ In brief, a weighted linear interpolation procedure, based upon the angular distance between each 10-2 stimulus location and the four surrounding 30-2 locations, was used to derive normal program 10-2 short wavelength perimetric thresholds. The short wavelength sensitivity of a given stimulus location for each diabetic patient was then compared to the mirror image location in the opposite horizontal hemifield of the same eye. Those stimulus locations that exhibited negative asymmetries greater than the confidence limits of the normal database were recorded. This analysis was developed in order to negate the effect of pre-receptoral absorption and light scatter on short wavelength sensitivity ${ }^{29-33}$ - that is, it was sensitive to defects in the shape of the SWAP "hill of vision" rather than being dependent upon absolute sensitivity. The area $\left(\right.$ degrees $\left.^{2}\right)$ of clusters (that is, three, or more, contiguous stimulus locations) of stimulus locations with negative asymmetries reaching statistical probability levels of $\mathrm{p}<0.05$ and $\mathrm{p}<0.005$ was calculated.

\section{Scanning laser tomography data}

The SLT images were analysed with the aid of custom software provided by Heidelberg Engineering (Heidelberg, Germany). The custom software produced image files of maximum reflectance intensity and of z-profile signal width. "Super pixel" values were averaged from each set of $4 \times 4$ pixels; the resulting resolution of the maximum reflectance intensity and the z-profile signal width image files was $64 \times 64$ super pixels. The z-profile signal width was measured at $50 \%$ of the maximum reflectance intensity following fitting of the z-profile with a 16th order polynomial. The data files were subsequently downloaded for custom analysis using the oedema mapping software utility (TView J Cassidy, JG Flanagan, and C Hudson). The maximum reflectance intensity maps were normalised in order to express reflectance intensity as a function of the range of intensity values within a given image. The normalisation procedure reduced the influence of variation in reflectance intensity between successive SLT images. ${ }^{134}$ The oedema index was then calculated for each pixel, such that:

Oedema index $\mathrm{x}_{\mathrm{i}}=\mathrm{SW}_{\mathrm{i}} / \mathrm{NI}_{\mathrm{i}}$

where $S W_{i}$ is the signal width $(\mu \mathrm{m})$ of the z-profile and $\mathrm{NI}_{\mathrm{i}}$ is the normalised maximum reflectance intensity (arbitrary units), at a given pixel, I. Consequently, the determination of the oedema index is a relative measurement and the derived values are in arbitrary units.

The mean oedema index provided a global summary that averaged the oedema index values of every pixel within the scan field. Manual alignment algorithms (TView) were used to establish the area of the oedema map that was common to each of the constituent SLT images; the common area was then used to calculate the mean oedema index for each visit and to analyse change in the oedema index between visits.

A Pearson correlation coefficient was undertaken on the data set of each diabetic patient to establish the correlation between change of visual function and change of the mean oedema index over the time course of the study. The following visual function parameters were used: (i) Regan logMAR visual acuity at $96 \%, 25 \%$, and $11 \%$ contrasts; (ii) mean deviation (MD) and corrected pattern standard deviation (CPSD) of conventional automated perimetry ${ }^{28}$; and (iii) SWAP cluster area of stimulus locations reaching statistical probability levels of $p<0.05$ and $p<0.005$. Correlation over the time course of the study resulted when the oedema index reduced and visual function improved, and/or when the oedema index increased and visual function deteriorated. Visual function was correlated with the mean oedema index derived for the $10^{\circ} \times 10^{\circ}$ and $20^{\circ} \times 20^{\circ}$ scan fields. A Bonferroni correction was applied to the correlations to correct for type I experimental error ( since multiple comparisons were undertaken on the mean oedema index to establish any significant correlation with the various parameters of visual function). Consequently, $\mathrm{p}<0.001$ was taken to indicate significant correlation.

\section{RESULTS}

Correlation of change of the mean oedema index and change of visual function over the time course of the study was found in 16 of the eligible 22 diabetic patients. One diabetic patient withdrew from the study after visit 3 and another patient was excluded from the correlation analysis owing to unreliable automated perimetry results. Thirteen diabetic patients exhibited significant correlation of the mean oedema index and at least one measure of visual function for the $10^{\circ} \times 10^{\circ}$ scan field and 10 patients showed correlation for the $20^{\circ} \times 20^{\circ}$ scan field. Seven patients demonstrated correlation for both scan fields.

For the $10^{\circ} \times 10^{\circ}$ scan field, four patients exhibited correlation with visual acuity (for one, or more, of the three Regan charts). Four patients exhibited correlation with SWAP (for one, or both, of the cluster area statistical levels), two of which also correlated with visual acuity. In addition, six patients exhibited correlation with MD. Six patients correlated with CPSD of conventional perimetry, four of which also correlated with MD. A single patient exhibited correlation with all visual function parameters-that is, visual acuity, MD, CPSD, and SWAP. 
Polient No $110^{\circ} \times 10^{\circ}$ field
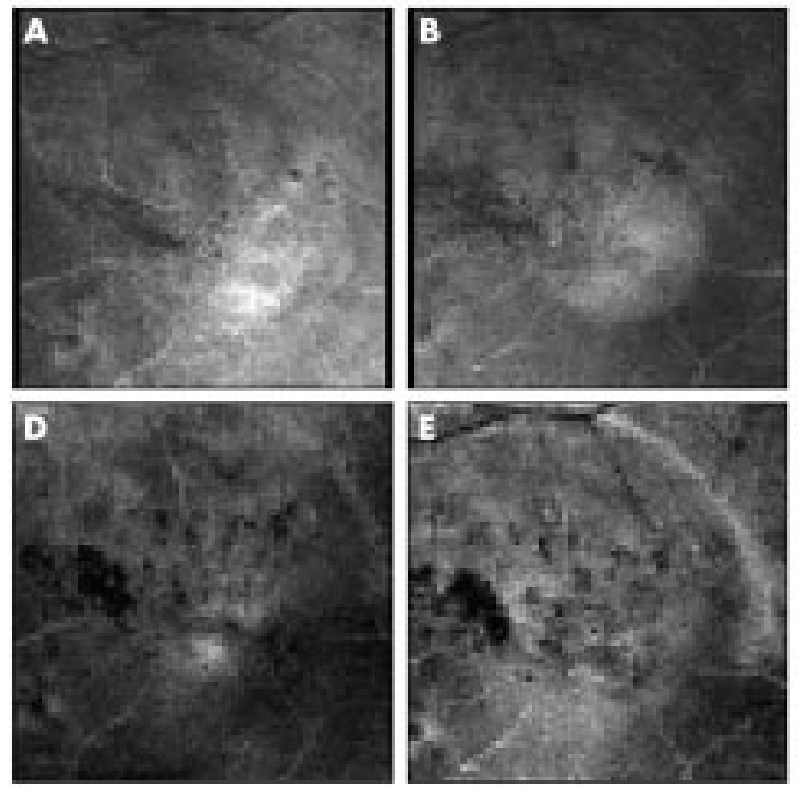

For the $20^{\circ} \times 20^{\circ}$ scan field, six patients exhibited correlation with visual acuity. Six patients correlated with SWAP, two of which also exhibited correlation with visual acuity. Three patients exhibited correlation with CPSD; all three of these patients also exhibited correlation with SWAP. In addition, two patients correlated with MD, both of which also correlated with CPSD and SWAP. A single patient exhibited correlation with all visual function parameters.

Two of the patients were selected for detailed description to illustrate a situation in which no correlation occurred (patient 1) and a situation in which significant correlation occurred with visual function (patient 23$)$. The oedema maps $\left(10^{\circ} \times 10^{\circ}\right.$ scan field) for diabetic patient 1 are shown in Figure 1 . The corresponding time courses of change in the mean oedema index and visual function are shown in Figure 2. Patient 1

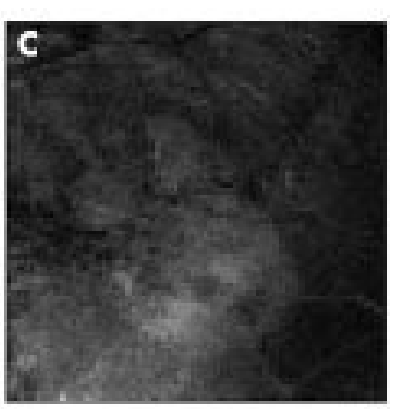

Figure 1 Oedema maps for diabetic patient 1 (left eye, $10^{\circ} \times 10^{\circ}$ scan field). The whiter the pixel the greater the magnitude of the oedema index $(A$; baseline. $B, C, D, E$, and $F$; 3 days, $1,2,4$, and 12 weeks post-treatment, respectively). The fovea is located approximately in the centre of each scan.

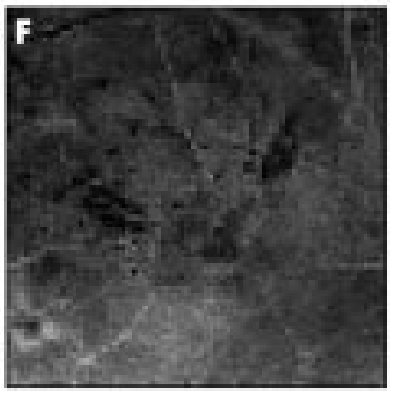

Figure 2 Time course of change in the mean oedema index value (upper left) for the $10^{\circ} \times 10^{\circ}$ and $20^{\circ} \times 20^{\circ}$ scan fields and the various parameters of visual function for diabetic patient 1 (Left eye). Upper right: Regan logMAR visual acuity (at $96 \%, 25 \%$, and $11 \%$ Weber contrasts). Lower left: MD and CPSD of conventional perimetry. Lower right: SWAP cluster volume (at statistical probability levels of $p<0.05$ and $\mathrm{p}<0.005)$. 
Patient No $2320^{\circ} \times 20^{\circ}$ field
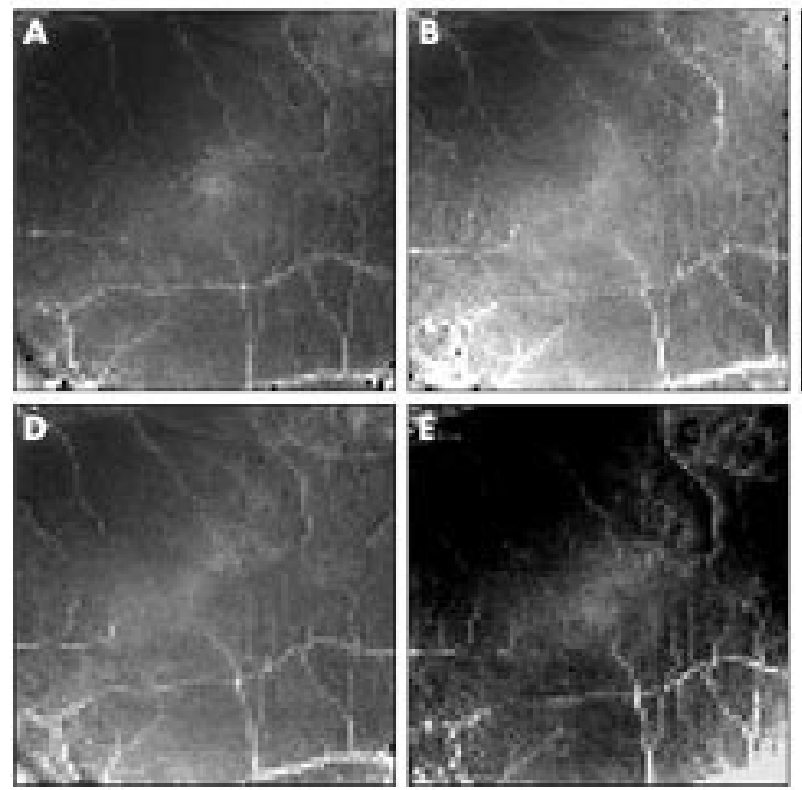

The oedema maps $\left(20^{\circ} \times 20^{\circ}\right.$ scan field $)$ for the baseline and subsequent visits of diabetic patient 23 are shown in Figure 3. The corresponding time courses of change in the mean oedema index $\left(10^{\circ} \times 10^{\circ}\right.$ and $20^{\circ} \times 20^{\circ}$ scan fields $)$ and the various parameters of visual function are shown in Figure 4. Patient 23 exhibited significant correlation of change of the mean oedema index for the $10^{\circ} \times 10^{\circ}$ scan field and SWAP cluster area $(\mathrm{p}<0.005)$. Patient 23 also exhibited significant correlation of the mean oedema index for the $20^{\circ} \times 20^{\circ}$ scan field and visual acuity (at $25 \%$ contrast), MD, and CPSD of conventional perimetry and SWAP cluster area $(p<0.005)$. The mean oedema index increased 3 days after laser treatment (Fig 4) and subsequently reduced relative to baseline. Those visual function parameters that demonstrated significant correlation deteriorated 3 days after laser treatment and/or improved relative to baseline.
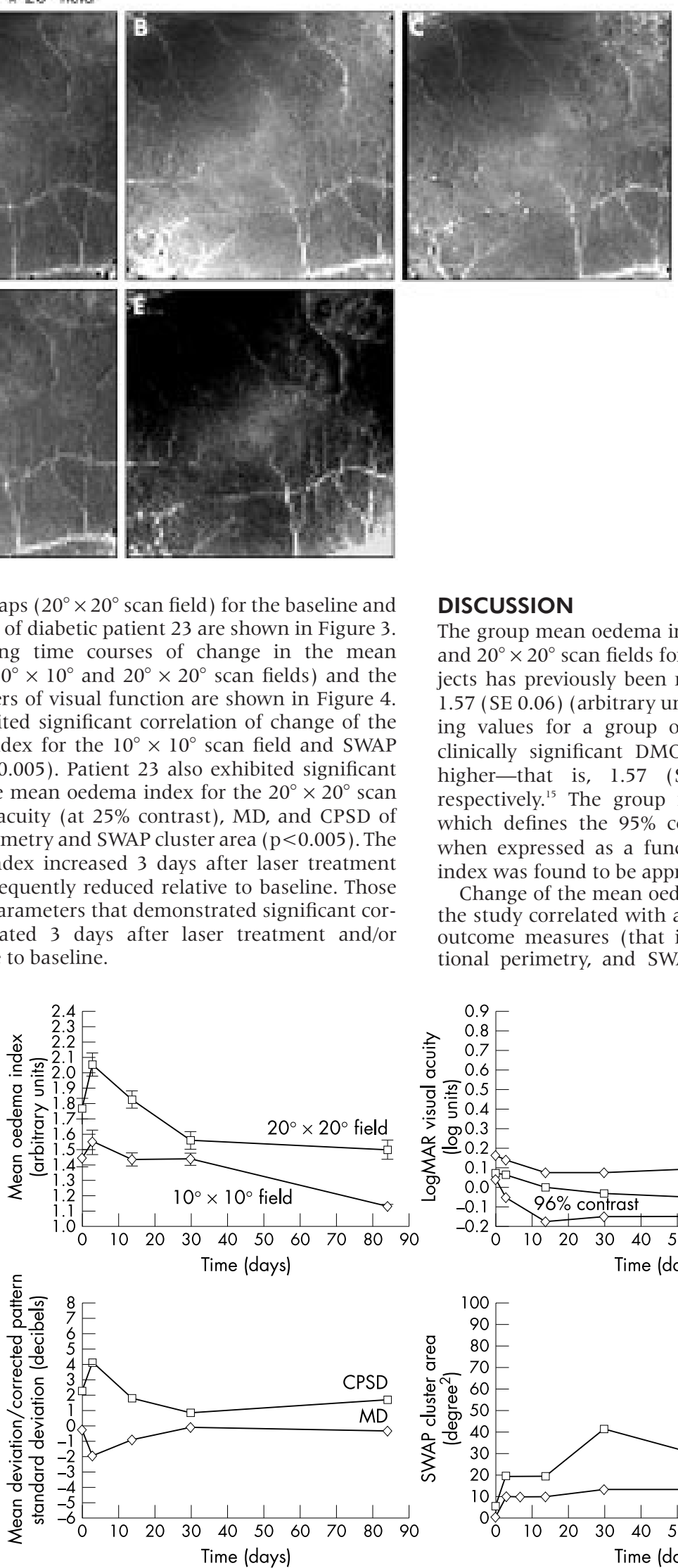

Figure 3 Oedema maps for diabetic patient 23 (Left eye, $20^{\circ} \times$ $20^{\circ}$ scan field). The whiter the pixe the greater the magnitude of the oedema index (A; baseline. B, C, D, and $\mathrm{E}_{;} 3$ days, 2,4 , and 12 weeks post-treatment, respectively). The patient failed to attend for the 1 week post-laser visit. The fovea is located approximately in the centre of each scan.

\section{DISCUSSION}

The group mean oedema index for all pixels within $10^{\circ} \times 10^{\circ}$ and $20^{\circ} \times 20^{\circ}$ scan fields for a group of 56 elderly normal subjects has previously been reported to be 1.21 (SE 0.04) and 1.57 (SE 0.06) (arbitrary units) respectively. ${ }^{15}$ The corresponding values for a group of 24 age matched patients with clinically significant DMO were found to be significantly higher-that is, 1.57 (SE 0.10) and 1.97 (SE 0.11) respectively. ${ }^{15}$ The group mean coefficient of repeatability, which defines the $95 \%$ confidence limits for repeatability, when expressed as a function of the group mean oedema index was found to be approximately $25 \% .^{15}$

Change of the mean oedema index over the time course of the study correlated with at least one of the three functional outcome measures (that is, logMAR visual acuity, conventional perimetry, and SWAP) in 16 of 22 eligible diabetic
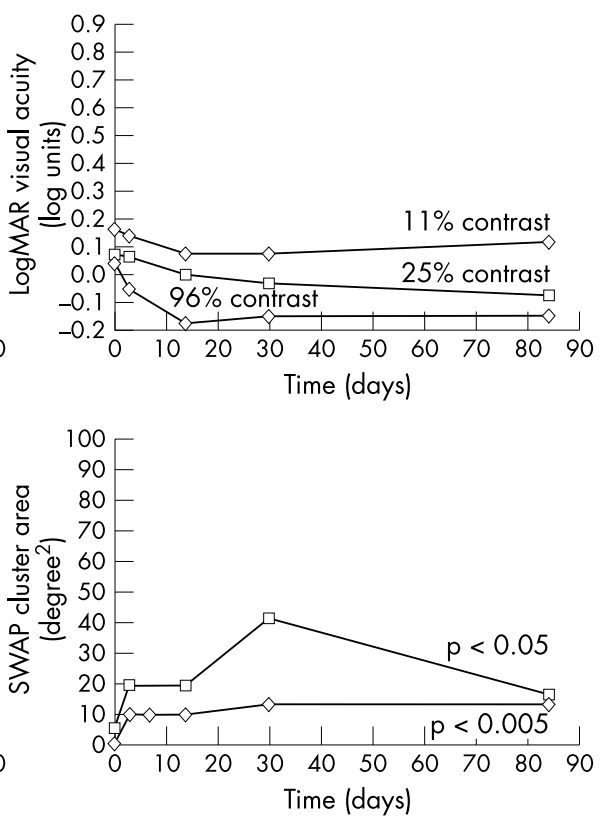

Figure 4 Time course of change in the mean oedema index value (upper left) for the $10^{\circ} \times 10^{\circ}$ and $20^{\circ} \times 20^{\circ}$ scan fields and the various parameters of visual function for diabetic patient 23 (Left eye). Upper right: Regan logMAR visual acuity (at $96 \%, 25 \%$, and $11 \%$ Weber contrasts). Lower left: MD and CPSD of conventional perimetry. Lower right: SWAP cluster volume (at statistical probability levels of $p<0.05$ and $\mathrm{p}<0.005)$. 
patients. Thirteen patients correlated for the $10^{\circ} \times 10^{\circ}$ scan field and 10 patients for the $20^{\circ} \times 20^{\circ}$ scan field; seven patients demonstrated correlation for both scan fields. Correlation of the mean oedema index and of visual function, however, was not found for every combination of parameters, or for every diabetic patient. This finding is consistent with the localised distribution of DMO and obvious differences in the time course of change of the various parameters.

The mean oedema index represents the average of the oedema index of every pixel within the scan field. All of the diabetic patients in this study exhibited localised areas of DMO. In some cases, localised change of the oedema index, although clearly visible on the oedema maps, could be obscured by the lack of change within the complete scan field (analogous to relying upon the MD index of automated perimetry to detect a focal relative visual field defect). Deterioration in visual acuity can result if a localised increase in the oedema index involves the fovea; such effects can occur alongside minimal change, or even a reduction, of the global mean oedema index (for example, patient 1). These findings emphasise the value of the oedema mapping technique. Reliance upon a global measure of retinal oedema in some situations will fail to detect subtle localised change. Similarly, techniques that rely upon a high level of interpolation (that is, values are inferred from closest nearby measured points rather than measured directly) to produce topographic maps of retinal thickening, such as optical coherence tomography ${ }^{35}$ and the retinal thickness analyser, ${ }^{36}$ will also fail to detect the true extent and magnitude of subtle localised change.

Obvious differences were also apparent in the time course of change of the various parameters. Grid laser photocoagulation typically resulted in an immediate localised sensitivity loss for conventional and short wavelength perimetric sensitivity ${ }^{11}$ whereas the resulting change of the mean oedema index occurred over a period of weeks (for example, patients 1 and 23). In addition, SWAP typically showed extensive and deep localised visual field loss in the diabetic patients before treatment ${ }^{10}$ and, consequently, was unable to reflect post-laser change of DMO because of dynamic range limitations imposed by the design of the perimetric stimulus. Interestingly, no relation was found between the photocoagulation treatment parameters and the various outcome measuresthe sample size was too small, however, for such a relation to be revealed.

To the best of our knowledge, no other study that has utilised an objective measure of retinal oedema has detected a transient increase of DMO following laser treatment (for example, patients 1 and 23). This can frequently be explained by an insufficient frequency of follow up. A transient increase of DMO involving the fovea is often clinically suspected following grid laser photocoagulation and is usually associated with a concomitant temporary reduction of visual acuity. Patients who exhibited an obvious post-laser reduction in visual acuity (for example, patient 1) showed an increase in localised oedema situated at the fovea (Fig 1). Resolution of the post-laser increase of the oedema index (for example, patient 23) was associated with a relative recovery of perimetric sensitivity and visual acuity (Fig 4). Indeed, we have previously reported a general recovery of perimetric sensitivity and visual acuity 3 months post-laser (relative to 3 days post-laser) in the same cohort of patients. ${ }^{11}$ The finding of a transient increase of DMO following treatment objectively documents the initial response of the retina to grid laser photocoagulation. By the same token, panretinal (or peripheral scatter) photocoagulation can result in a more obvious transient reduction of visual acuity that is associated with the spontaneous development of macular oedema or the worsening of pre-existing DMO. ${ }^{37}$

Uncorrected refractive error can result in broadening of the z-profile ${ }^{913}$ and, consequently, artefactual increase of the oedema index. The spherical refractive error component was corrected for every patient by appropriate adjustment of the refraction on the HRT control panel. Cylindrical refractive error was not corrected; however, only one diabetic patient had astigmatism greater than 0.75 dioptres and the study essentially measured change in the oedema index over time. Consequently, the impact of astigmatism on the outcome of the study was minimal.

In summary, this study has objectively and non-invasively documented change in the magnitude and distribution of DMO for patients undergoing grid laser treatment and has established the relation of this change to that of visual function. Correlation of change over time in the two main parameters of the study - that is, visual function and the SLT derived oedema index, occurred in 16 patients. Significant correlation did not occur in all possible situations because, firstly, laser photocoagulation typically resulted in an immediate loss of perimetric sensitivity whereas the oedema index changed over a period of weeks. Secondly, localised oedema failed to impact upon visual acuity or letter contrast sensitivity when situated extrafoveally. Thirdly, localised change of the oedema index was often obscured by the lack of change within the rest of the scan field. Furthermore, the study has revealed new information about the progress of DMO following laser treatment. The finding of a transient increase of DMO objectively documents the initial response of the retina to grid laser photocoagulation. In addition, the protocol described in the paper can be used in the future to effectively evaluate new treatment strategies for the amelioration of DMO.

\section{ACKNOWLEDGEMENTS}

We thank Dr Gerhard Zinser of Heidelberg Engineering for providing the software to derive the z-profile signal width and James Cassidy for the development of TView.

Grant support: This work was funded by a postdoctoral fellowship from Diabetes UK (awarded to Dr Hudson) and by a research grant from Alcon Laboratories Inc (awarded to Drs Flanagan and Hudson).

Proprietary interest: Cc 5.

Authors' affiliations

C Hudson, J G Flanagan, G S Turner, H C Chen, L B Young,

D McLeod, University Department of Ophthalmology, Royal Eye Hospital, Manchester M13 9WH, UK

C Hudson, J G Flanagan, School of Optometry, University of Waterloo, Waterloo, Ontario N2L $3 \mathrm{Gl}$, and Department of Ophthalmology, University of Toronto, Toronto, Ontario M5T 2S8, Canada

\section{REFERENCES}

1 Klein R, Klein BEK, Moss SE, et al. The Wisconsin epidemiologic study of diabetic retinopathy. IV. Diabetic macular edema. Ophthalmology 1984:91:1464-74.

2 Klein R, Moss SE, Klein BEK, et al. The Wisconsin epidemiologic study of diabetic retinopathy. IX. The incidence of macular edema. Ophthalmology 1989;96:1501-10.

3 Klein R, Klein BEK, Moss SE, et al. The Wisconsin epidemiology study of diabetic retinopathy. XVII. The 14 year incidence and progression of diabetic retinopathy and associated risk factors in type I diabetes. Ophthalmology 1998;105:1799-815.

4 ETDRS Research Group. Early photocoagulation for diabetic retinopathy. ETDRS Report Number 9. Ophthalmology 1991;98:766-85.

5 Kinyoun J, Barton F, Fisher M, et al . Detection of diabetic macular edema. Ophthalmoscopy versus photography. ETDRS report number 5 Ophthalmology 1989;96:746-51.

6 Ferris FL, Patz A. Macular edema. A complication of diabetic retinopathy. Surv Ophthalmol 1984;28:452-61.

7 Hudson C, Flanagan JG, McLeod D. A clinical vision science perspective of the management of diabetic macular oedema. Excerpta Medica. Focus on Diabetic Retinopathy. 2001;8:4-9.

8 Bresnick GH. Diabetic macular edema. Ophthalmology 1986:93:989-97.

9 Bartsch DW, Intaglietta M, Bille JF, et al. Confocal laser tomographic analysis of the retina in eyes with macular hole formation and other focal macular diseases. Am J Ophthalmol 1989;108:277-87.

10 Hudson C, Flanagan JG, Turner GS, et al. Short-wavelength sensitive visual field loss in patients with clinically significant diabetic macular oedema. Diabetologia 1998;41:918-28. 
11 Hudson C, Flanagan JG, Turner GS, et al. Influence of laser photocoagulation for clinically significant diabetic macular oedema (DMO) on short-wavelength and conventional automated perimetry. Diabetologia 1998;41:1283-92.

12 McCreesh BM, Hudson C, Silvestri G, et al. Short-wavelength automated perimetry in diabetic patients with non-oedematous retinopathy and with pre-treatable macular oedema. [ARVO abstract] Invest Ophthalmol Vis Sci 1999;40:S308 (Abstract no 1629)

13 Hudson C, Flanagan JG, Turner GS, et al. Scanning laser tomography Z-profile signal width as an objective index of retinal thickening due to macular oedema. Br J Ophthalmol 1998;82:121-30.

14 Bartsch D-U, Freeman WR. Axial intensity distribution analysis of the human retina with a confocal scanning laser tomograph. Exp Eye Res 1994;58:161-73.

15 Flanagan JG, Hudson C. A novel analysis of scanning laser tomography images for the objective, quantitative mapping of retinal edema. Retina (submitted)

16 Hudson C, Flanagan JG, Turner GS, et al. Scanning laser derived edema index topographic maps: correlation with visual function assessment in patients undergoing laser photocoagulation for clinically significant diabetic macular edema. In: Wall M, Wild JM, eds. Perimetry update 1998-9. Proceedings of the XIllth International Perimetric Society Meeting. The Hague, The Netherlands: Kugler Publications; 1999:503-10

17 Early Treatment Diabetic Retinopathy Study Research group. Treatment techniques and clinical guidelines for photocoagulation of diabetic macular edema. ETDRS Report Number 2. Ophthalmology 1987;94:761-74.

18 Chylack LT, Wolfe JK, Singer DM, et al. The lens opacities classification system III. Arch Ophthalmol 1993;111:831-6.

19 Heijl A, Lindgren G, Olsson J. The effect of perimetric experience in normal subjects. Arch Ophthalmol 1989;107:81-6.

20 Bron AJ, Sparrow J, Brown NAP, et al. The lens in diabetes. Eye 1993;7:260-75

21 Elliott DB, Bullimore MA, Bailey IL. Improving the reliability of the Pelli-Robson contrast sensitivity test. Clin Vis Sci 1991;6:471-5.

22 Early Treatment Diabetic Retinopathy Study Research Group Photocoagulation for diabetic macular edema. ETDRS Report Number 1 Arch Ophthalmol 1985;103:1796-806.

23 Early Treatment Diabetic Retinopathy Study Research Group. Early photocoagulation for diabetic retinopathy. ETDRS Report Number 9. Ophthalmology 1991;98:766-85.
24 Early Treatment Diabetic Retinopathy Study Research Group. Focal photocoagulation treatment for diabetic macular oedema. ETDRS Report Number 19. Arch Ophthalmol 1995;113:1144-55

25 Davies EG, Petty RG, Kohner EM. Long term effectiveness of photocoagulation for diabetic maculopathy. Eye 1989;3:764-7.

26 Sample PA, Johnson CA, Haegerstrom-Portnoy G, et al. Optimum parameters for short-wavelength automated perimetry. J Glaucoma 1996:5:375-83

27 Hudson C, Wild JM, O'Neill EC. Fatigue effects during a single session of automated static threshold perimetry. Invest Ophthalmol Vis Sci 1994:35:268-80.

28 Heijl A, Lindgren $G$, Olsson J. A package for the statistical analysis of visual fields. In: Greve EL, Heiil A, eds. Seventh International Visual Field Symposium. Doc Ophthalmol Proc Ser 49. Dordrecht: Martinus Nijhoff/ Dr W Junk; 1987: 153-68.

29 Sample PA, Esterson FD, Weinreb RN, et al. The ageing lens: In vivo assessment of light absorption in 84 human eyes. Invest Ophthalmol Vis Sci 1988;29:1306-11.

30 Sample PA, Esterson FD, Weinreb RN. A practical method for obtaining an index of lens density with an automated perimeter. Invest Ophhalmol Vis Sci 1989;30:786-7.

31 Moss ID, Wild JM, Whitaker DJ. The influence of cataract on blue-on-yellow perimetry. Invest Ophthalmol Vis Sci 1995:36:764-73.

32 Wild JM, Hudson C. The attenuation of blue-on-yellow perimetry by the macular pigment. Ophthalmology 1995;102:911-17.

33 Hudson C, Wild JM. The influence of pre-receptoral absorption on blue/yellow automated perimetry. In: Mills RP, ed. Perimetry update 1992-93. Proceedings of the Xth International Perimetric Society Meeting. Amsterdam, New York: Kugler Publications, 1993:45 1-457.

34 Eikelboom RH, Cooper RL, Barry CJ. A study of variance in densitometry of retinal nerve fiber layer photographs in normals and glaucoma suspects. Invest Ophthalmol Vis Sci 1990;31:2373-83.

35 Puliafito CA, Hee MR, Lin CP, et al. Imaging of macular disses with optical coherence tomography. Ophthalmology 1995;102:217-29.

36 Shahidi M, Ogura Y, Blair NP, et al. Retinal thickness change after focal laser treatment of diabetic macular oedema. $\mathrm{Br} J$ Ophthalmol 1994:78:827-30.

37 Meyers MS. Macular edema after scatter laser photocoagulation for proliferative diabetic retinopathy. Am J Ophthalmol 1980;90:210-16.

\section{Video Reports (www.bjophthalmol.com)}

- Capsule staining and mature cataracts: a comparison of indocyanine green and trypan blue dyes. D F Chang

- Pearls for implanting the Staar toric IOL. D F Chang

- An intraocular steroid delivery system for cataract surgery. D F Chang

- Evaluation of leucocyte dynamics in mouse retinal circulation with scanning laser ophthalmoscopy. H XU, A Manivannan, G Daniels, J Liversidge, P F Sharp, J V Forrester, IJ Crane

- Dipetalonema reconditum in the human eye. T Huynh, J Thean, R Maini

- Surgical revision of leaking filtering blebs with an autologous conjunctival graft. $K$ Taherian, A Azuara-Blanco

- Thixotropy: a novel explanation for the cause of lagophthalmos after peripheral facial nerve palsy. M Aramideh, JHTM Koelman, PP Devriese, F VanderWerf, JD Speelman 